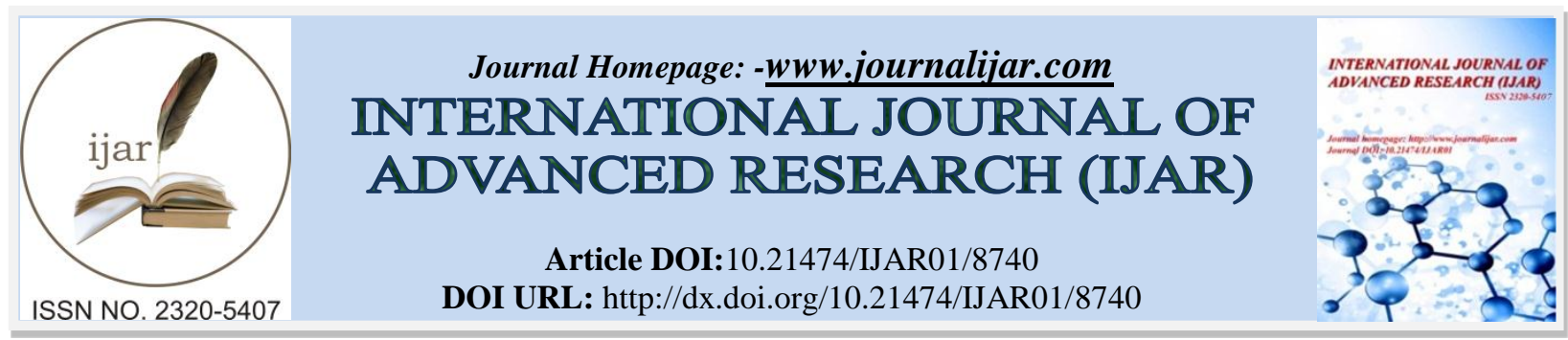

RESEARCH ARTICLE

\title{
ACUTE COCAINE MYOCARDITIS IN A YOUNG HEALTHY PATIENT.
}

\author{
Dr. Amine Qat, Dr. Zakaria El Arbaoui, Dr. Youness Halabi Kettani, Pr. Nawal Doghmi and \\ Pr. Mohamed Cherti. \\ Department of Cardiology B, University Hospital of Rabat, Morocco.
}

\section{Manuscript Info}

Manuscript History

Received: 19 January 2019

Final Accepted: 21 February 2019

Published: March 2019

\begin{abstract}
Cardiac arrhythmia and acute myocardial ischemia are the most common causes of cocaine-induced sudden cardiac death. However, cocaine induced myocarditis is uncommon. We report a case of a 22year-old male with no significant medical history who presented with palpitations and atypical chest pain. Interrogation showed recreational drug use, particularly cocaine use through nasal inhaling. ECG was normal, however cardiac enzymes were elevated, and transthoracic echocardiography showed regional wall abnormalities with a preserved left ventricular ejection fraction $(\mathrm{LVEF}=60 \%)$. Coronary angiography revealed no significant obstructive, and Cardiac MRI, using delayed myocardial enhancement sequence, was consistent with myocarditis.
\end{abstract}

Copy Right, IJAR, 2019,. All rights reserved.

\section{Introduction:-}

A 22-year-old male patient, with a history of cigarette and cannabis smoking, and also occasional drinking, presented to the emergency department with a sudden onset of palpitations associated with atypical chest pain. After thorough questioning, the patient admitted to inhaling cocaine intranasally a day before (no flu-like symptoms were noted in the prior days).

Physical exam and electrocardiogram (figure 1) were normal. However, cardiac biomarkers were elevated (Cardiac Troponin was 400 times higher than normal value).

Also, transthoracic echocardiography showed regional wall abnormalities with severe hypokinesia of the apex and apical segments of anterior and antero-septal walls, with a preserved left ventricular ejection fraction (LVEF: 60\%). Coronary angiography revealed no significant obstructive coronary artery disease (Figure 2; Figure 3).

However, the patient underwent (one week after the onset of the symptomatology) a Cardiac Magnetic Resonance Imaging (MRI), and using the Delayed myocardial enhancement sequence (10 minutes following the administration of a Gadolinium based contrast agent), it showed an intramyocardial delayed contrast accumulation in anterior, inferior and antero-lateral walls, with no motion wall abnormalities, non-dilated non-hypertrophied LV with a preserved $\mathrm{EF}=60 \%$. The diagnosis was consistent with myocarditis (Figure 4; Figure 5; Figure 6).

Thus, the patient was treated with low dose of beta-blockers (in order to prevent arrhythmia), with a good evolution on both biological (cardiac troponin fall) and clinical features (no recurrence of palpitations nor atypical chest pain). 
Also, transthoracic echocardiography follow-up was done 2 weeks later and showed normal wall motion of the left ventricle.

\section{Discussion:-}

Cocaine was shown to induce myocarditis, either through elevated levels of catecholamines, creating myocardial necrosis and local immune reaction, or from the induction of eosinophilic myocarditis [1].

Virmani et al [2] in their autopsy study have confirmed cocaine-induced myocarditis as an important cause of death in cocaine abuse. In cocaine-induced myocarditis, small foci of myocytes necrosis are scattered in different areas of the heart associated with infiltrations of lymphocytes. Under electron microscope, vacuolization of the sarcoplasmic reticulum and loss of myofibrils are found in the lesion. The mononuclear cellular infiltrations can be associated with various degrees of interstitial fibrosis. Biopsy specimen findings that include myocytes necrosis along with mononuclear cellular infiltration may be a result of acute cocaine-induced direct cardiotoxicity [3, 4].

Lymphocytic myocarditis serves also as an anatomic substrate for cardiac arrhythmia and ultimately develops a permanent dilated cardiomyopathy [3].

Myocardial lesions caused by cocaine-induced myocarditis might produce ventricular arrhythmias, either in the acute phase (as a result of increased excitability) or after recovery (scar-mediated from myocardial fibrosis) [5].

In our case, the 22 year old patient's ECG showed a NSTEMI with normal coronarography.

However cardiac MRI showed an intramyocardial delayed contrast accumulation in anterior, inferior and anterolateral walls, with no oedema and no motion wall abnormalities on the LV and a preserved EF, consistent with myocarditis.

Thus the importance of cardiac MRI, using Delayed Myocardial Enhancement imaging technique, which helps differentiate between an ischemic (subendocardial delayed enhancement) and a non-ischemic etiology (midwall or epicardial delayed enhancement).

\section{Conclusion:-}

The recognition of cocaine induced myocarditis is crucial for optimal management. A previously healthy young person presenting with cardiac type chest pain should be asked about cocaine use. Contrast cardiac MRI is the key to help differentiating between ischemic and non-ischemic causes of cardiomyopathy, especially after coronary angiography reveals no significant coronary artery disease.

\section{Disclosure:}

The author reports no conflicts of interest in this work. 


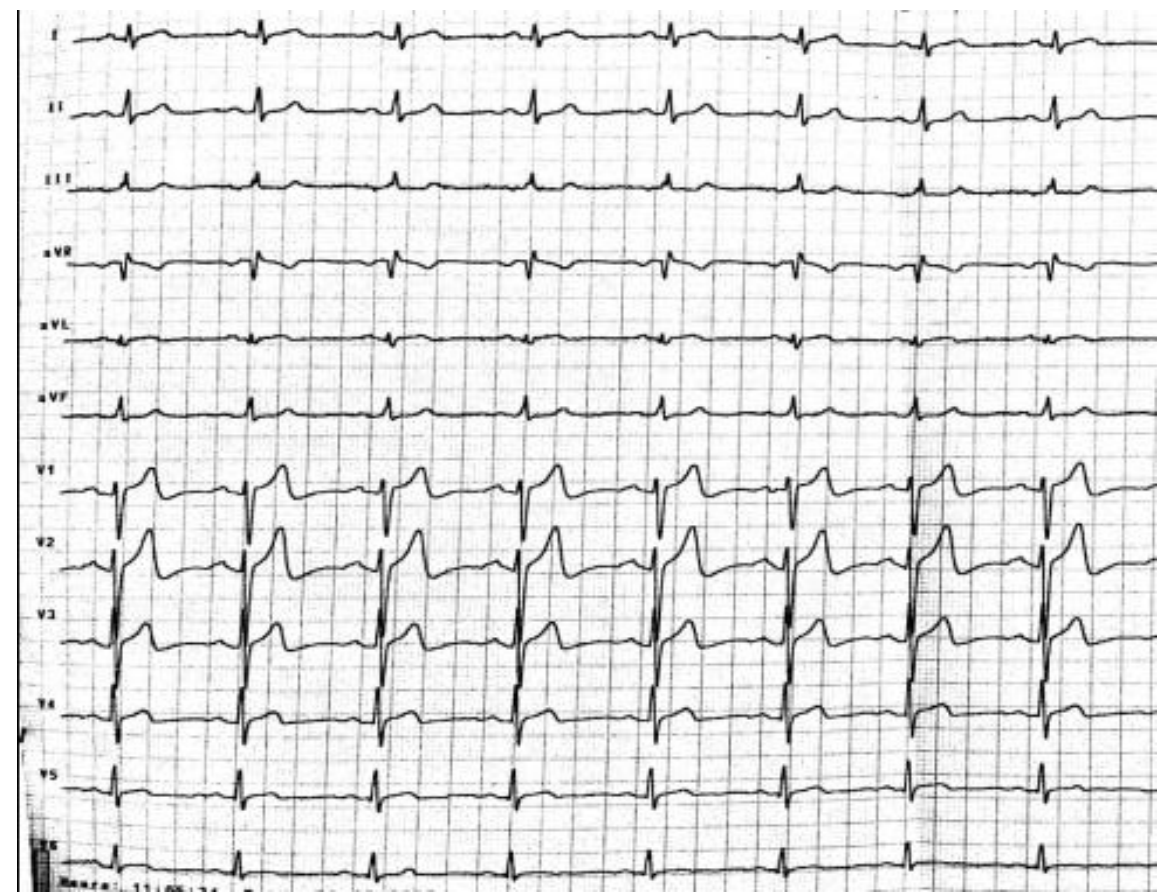

Figure 1:-Electrocardiogram of the patient, showing a sinus regular rhythm at $63 \mathrm{bpm}$, with no repolarization nor conduction abnormalities

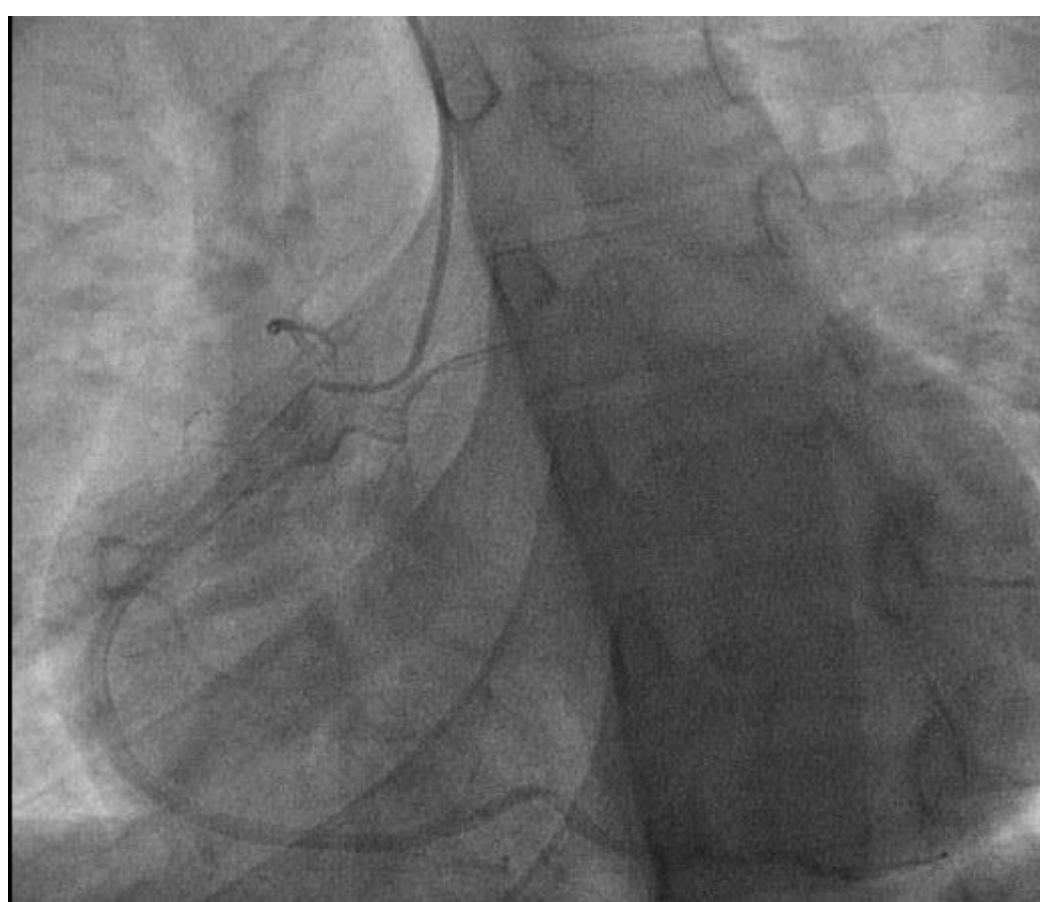

Figure 2:-LAO 30 View - Right coronary artery of a good caliber, with no stenosis 
Figure 3:-Cranial 40 view - Visualization of the LAD with a good caliber, free of stenosis, gives off normal diagonal branches.

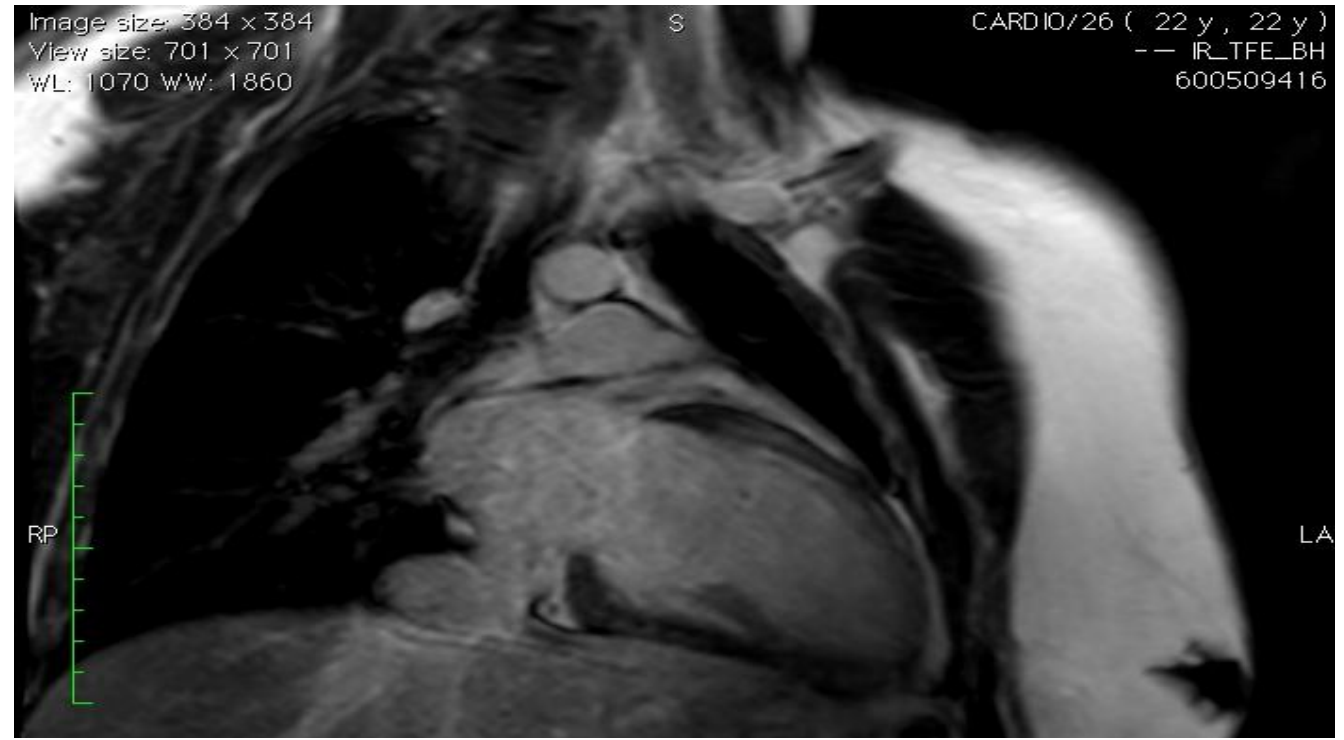

Figure 4:-Delayed myocardial enhancement MRI in a 2-Chambers view showing an intramyocardial delayed contrast accumulation in anterior and inferior walls. 


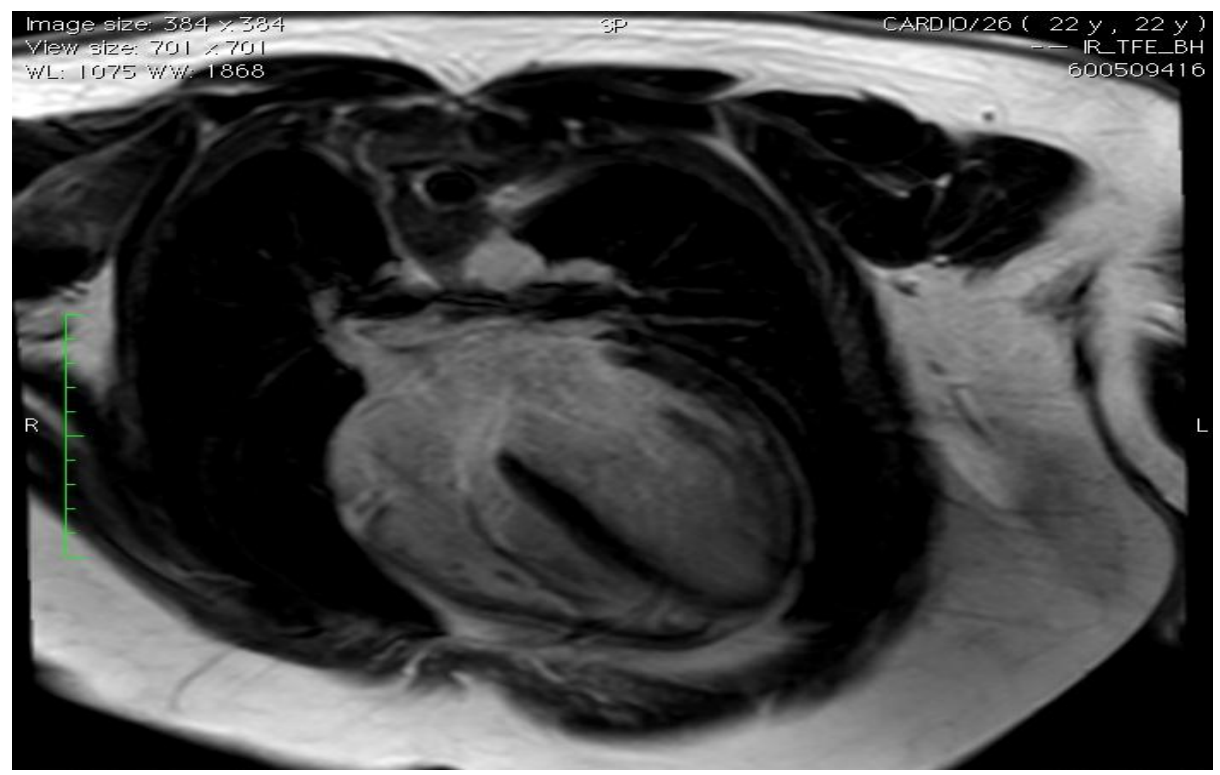

Figure 5:-Delayed myocardial enhancement MRI in a 4-Chambers view showing an intramyocardial delayed contrast accumulation in antero-lateral wall.

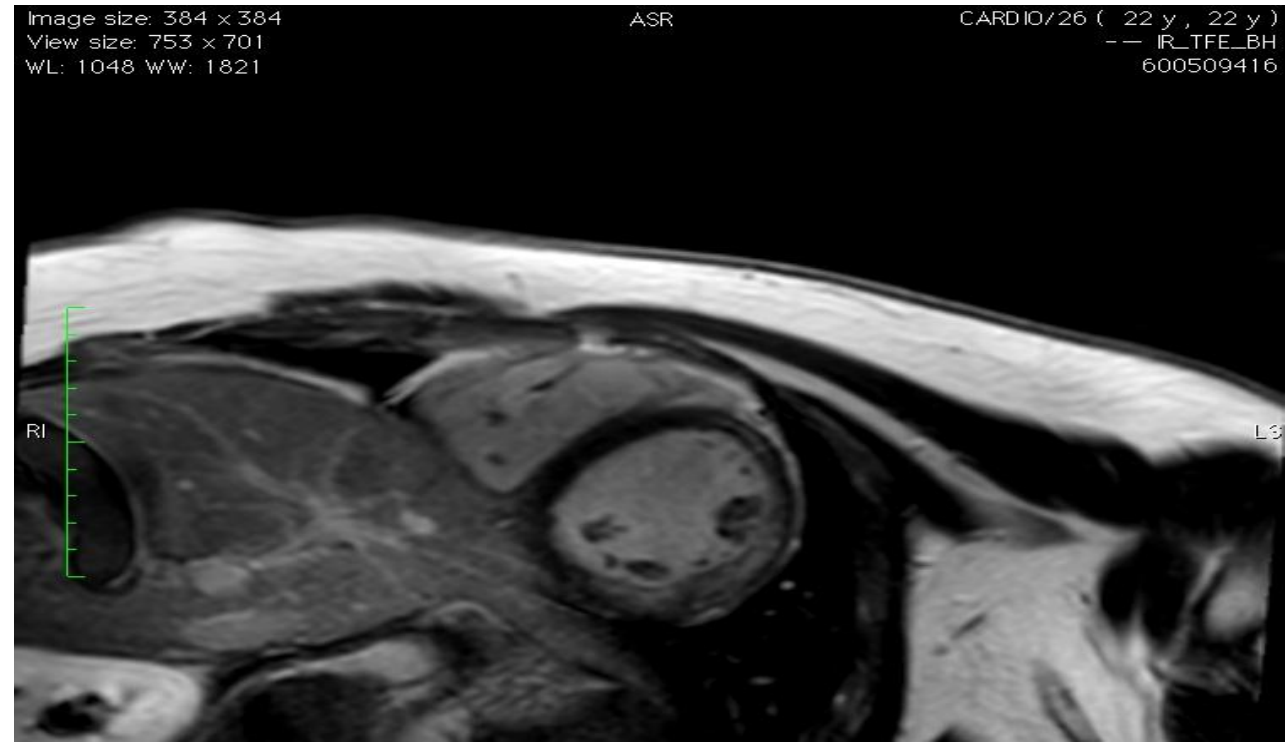

Figure 6:-Delayed myocardial enhancement MRI in a short axis view showing an intramyocardial delayed contrast accumulation in anterior, inferior and antero-lateral walls.

\section{References:-}

1. Isner JM, Estes NA III, Thompson PD, et al. Acute cardiac events temporally related to cocaine,abuse. N Engl J Med 1986;315:1438-43.

2. Virmani R, Robinowitz M, Smialek JE, Smyth DF. Cardiovascular effects of cocaine: an autopsy study of 40 patients. American Heart Journal. 1988; 115(5): 1068-1076.

3. Kloner RA, Hale S, Alker K, Rezkalla S. The effects of acute and chronic cocaine use on the heart. Circulation. 1992;85(2):407-419

4. Peng SK, French WJ, Pelikan PC. Direct cocaine cardiotoxicity demonstrated by endomyocardial biopsy. Archives of Pathology and Laboratory Medicine. 1989;113(8):842-845

5. Friedman RA, Kearney DL, Moak JP, Fenrich AL, Perry JC. Persistence of ventricular arrhythmia after resolution of occult myocarditis in children and young adults. J Am Coll Cardiol 1994;24:780-3. 